\title{
Diagnóstico Laboratorial do C arcinoma Medular de Tiróide: Calcitonina Basal e Testes de Estímulo
}

\section{RESUMO}

A calcitonina é um hormônio secretado pelas células parafoliculares tiroideanas. O diagnóstico de carcinoma medular de tiróide (CMT) pode ser suspeitado a partir do encontro de níveis aumentados de calcitonina basal ou após testes de estímulos específicos. Neste sentido, a calcitonina caracteriza-se como um importante marcadortumoral para o diagnóstic o e seguimento de pacientesportadores de CMT. Neste a rtigo, revisamos alguns tópicos referentes à utilidade da dosagem de calcitonina basal em pacientes portadores de nódulos de tiróide para rastreamento de CMT. Comentamos a respeito dos principa is testes de estímulo para secreção de calcitonina disponíveis e finalizamos com algumas observações sobre os ensaios para dosagem de calcitonina. (Arq Bras Endocrinol Metab 2003;47/5:529-533)

Descritores: Calcitonina; Carcinoma medular de tiróide; Testes de estímulo

\begin{abstract}
Biochemical Diagnosis of Medullary Thyroid Carc inoma: Basal Calc itonin and Provocative Tests.

Calcitonin is a homone sec reted by the parafollic ular thyroid cells. Diagnosis of medullary thyroid carcinoma (MTC) should be considered when high basal calcitonin levels or high calcitonin levels after specific provocative test are found. In this context, calcitonin is considered a relevant tumor marker for diagnosis and follow-up of MTC. In this manuscript, we review some issues related to the usefulness of measuring basal calcitonin in patients with thyroid nodules. The current available provocative tests for calcitonin a re discussed. Finally, we comment on some aspects related to the assays for calcitonin measurement. (Arq Bras Endoc rinol Metab 2003;47/5:529-533)
\end{abstract}

Keywords: Calcitonin; Medullary thyroid carcinoma; Provoca tive tests

A CALCITONINA É UM HORMÔNIO POLIPEPTÍDICO composto por 32 aminoácidos, cuja secreção é originária preponderantemente das células $C$ ou parafoliculares tiroideanas. Do ponto de vista terapêutico, tem aplicações para o controle da hipercalcemia e também como agente antireabsortivo ósseo. Do ponto de vista diagnóstico, a calcitonina representa um marcador tumoral para o diagnóstico e seguimento de pacientes portadores de carcinoma medular de tiróide (CMT) (1).

O CM T é um tumor de células parafoliculares tiroideanas, respondendo por cerca de 4 a 10\% dos carcinomas de tiróide. Cerca de $25 \%$ destes casos são familiares (CMT familiar isolado [CMTF] ou fazendo parte de neoplasias endócrinas múltiplas [ $N E M$ ] tipo $2 \mathrm{~A}$ ou $2 \mathrm{~B}$ ) e mutações específicas do proto-oncogene R ET foram identificadas em 85 a $97 \%$ das famílias afetadas. Desta forma, em famílias sabidamente portadoras de CMT, a pesquisa de mutações do RET deve ser inicialmente realizada, com a fina- revisão

\author{
Omar M. H auache \\ J osé Gi lberto H . Viei ra \\ R ui M.B. Maciel
}

Setor de Endocrinologia, Fleury Centro de M edicina Diagnóstica; e Disciplina de Endocrinologia da Escola Paulista de M edicina, U niversidade Federal de São Paulo, São Paulo, SP. 
lidade de identificação de indivíduos em risco de ter a doença. A maioria destas mutações situa-se em locais bem definidos do proto-oncogene RET (hot spots) e está associada a fenótipos distintos. $N$ estes casos, o estímulo anual de secreção de calcitonina com pentagastrina e/ ou cálcio deve ser reservado apenas para determinados casos portadores de mutação do RET, no sentido de auxiliar a decisão do momento para indicação de tiroidectomia total. Com a possibilidade do teste molecular, CMTF pode ser diagnosticado precocemente, mesmo em fase présintomática, resultando em taxas de cura elevadas nos indivíduos afetados $(2,3)$.

Já nos pacientes portadores de CMT na forma esporádica, identificam-se mutações ativadoras do RET em apenas 5 a 7\% dos casos estudados. Pacientes com CMT esporádico muitas vezes têm sua apresentação inicial caracterizada pelo achado de nódulos de tiróide ao ultra-som ou, mais raramente, doença metastática pode ser evidenciada logo no início da investigação. Geralmente, estes tumores esporádicos são solitários e localizados na porção média ou superior de um lobo tiroideano. A maioria destes pacientes encontra-se entre a 5 a e a 7 a . décadas de vida, e muitos têm linfadenopatia cervical (4). A coexistência de outras características de NEM 2A (feocromocitoma, hiperparatiroidismo primário) ou NEM 2B (feocromocitoma, neuromas, hábito marfanóide) com um nódulo de tiróide deve aumentar o grau de suspeita para CMT.

\section{Dosagem de calcitonina basal em pacientes portadores de nódulo de tiróide}

Como o estadio da doença é o fator prognóstico mais relevante, preconiza-se que pacientes com CMT esporádico devam ser identificados numa fase ainda precoce da doença. D iversos estudos foram publicados nos últimos anos abordando a utilidade da medida rotineira de calcitonina com a finalidade de permitir um diagnóstico precoce de CMT em pacientes com nódulos de tiróide. Estes estudos encontraram uma prevalência de CM T de 0,6 a 1,4\% em pacientes portadores de bócio nodular. Baseado nestes resultados, a sugestão de inclusão de medida de calcitonina na avaliação rotineira de nódulos de tiróide passou a ser amplamente discutida (5-10).

$\mathrm{N}$ este sentido, a pergunta a ser formulada é a seguinte: qual a utilidade ou o quanto é útil dosar calcitonina com o intuito de se fazer um diagnóstico préoperatório de CM T esporádico? T al resposta reside no conhecimento de que a calcitonina é um marcador sensível e específico para o diagnóstico de hiperplasia de células C e CM T. Diversos estudos publicados nos últimos 10 anos sugerem que a medida de calcitonina basal em pacientes com doença nodular tiroideana deve ser considerada levando-se em conta o achado de CM T em até $41 \%$ dos pacientes que apresentam níveis de calcitonina basal elevados (8).

0 custo para dosagem de calcitonina também é um fator importante quando pensamos na relação custo-benefício associada à abordagem de dosar calcitonina em pacientes portadores de nódulos tiroideanos. N os Estados U nidos, uma dosagem de calcitonina custa cerca de 100 dólares (8). No estudo de $\mathrm{N}$ iccoli e cols. (7), no qual 1.167 pacientes portadores de um ou mais nódulos de tiróide foram estudados com dosagem basal de calcitonina sérica por método imunométrico, 16 casos foram diagnosticados como portadores de CMT. Desta forma, o custo para detecção destes casos foi de 5.800 dólares por caso. A pesar deste valor ser alto, a identificação de CMT como nódulo palpável de tiróide está associada com percentual elevado de metástases, implicando em tratamento adicional (8). Assim, o custo do rastreamento inicial com dosagem de calcitonina deve ser avaliado em comparação com a necessidade de diversas cirurgias, radioterapia e quimioterapia, esta última muitas vezes utilizada em pacientes com doença metastática.

Além disso, os níveis pré-operatórios de calcitonina correlacionam-se significativamente com o tamanho do tumor e com a possibilidade de metástases à distância. Cohen e cols. (11), utilizando método imunorradiométrico (IRMA) para dosagem de calcitonina, demonstraram que um nível basal pré-operatório de calcitonina superior a $1.000 \mathrm{pg} / \mathrm{mL}$ correspondeu à mediana de tamanho tumoral de $25 \mathrm{~mm}$ e associou-se ao encontro de metástases à distância em 10,5\% dos casos, enquanto que casos com níveis inferiores a $1.000 \mathrm{pg} / \mathrm{mL}$ apresentaram tumores com mediana de $7 \mathrm{~mm}$ e metástases à distância em apenas 3,3 \% dos casos. N os casos com calcitonina menor que $100 \mathrm{pg} / \mathrm{mL}$, a mediana de tamanho tumoral correspondeu a $3 \mathrm{~mm}$. Neste mesmo trabalho, os autores descreveram que níveis pré-operatórios de calcitonina basal inferiores a $50 \mathrm{pg} / \mathrm{mL}$ correlacionaram-se com normalização pósoperatória em $97,8 \%$ dos casos estudados.

$\mathrm{N}$ o período de setembro de 2001 a setembro de 2002, realizamos 1.124 dosagens de calcitonina basal no Fleury - Centro de M edicina D iagnóstica, utilizando um ensaio imunoquimioluminométrico de dupla identificação que detecta a molécula de calcitonina intacta, com nível de deteç̧ão a partir de $2 \mathrm{pg} / \mathrm{mL}$ (12). A maioria destas dosagens foi realizada em muIheres $(n=896)$. Do total destas 1.124 dosagens, $1.051(93,5 \%)$ apresentaram calcitonina inferior a 
$10 \mathrm{pg} / \mathrm{mL}$. Nível inferior a $5 \mathrm{pg} / \mathrm{mL}$ foi encontrado em 986 indivíduos (87,5\%). M ais além, gostaríamos de destacar que 771 dosagens (68,5\%) eram inferiores a $2 \mathrm{pg} / \mathrm{mL}$. D esta forma, apenas 73 dosagens (6,5\%) foram superiores a $10 \mathrm{pg} / \mathrm{mL}$. A mediana do valor de calcitonina para ambos os sexos, descontando-se os valores indetectáveis (menor que $2 \mathrm{pg} / \mathrm{mL}$ ), foi de $4 \mathrm{pg} / \mathrm{mL}$ (intervalo de confiança de $95 \%$ ). Nossos dados são semelhantes aos dados relatados em estudo recente (13), que revelou valores basais de calcitonina inferiores a $1 \mathrm{pg} / \mathrm{mL}$ em $87 \%$ dos homens normais e em $93 \%$ das mulheres normais estudadas $(n=340)$. N este mesmo estudo (13), em 48 pacientes portadores de doenças tiroideanas benignas, a calcitonina foi menor que $1 \mathrm{pg} / \mathrm{mL}$ em $87,5 \%$ dos casos, com concentração máxima de $7,9 \mathrm{pg} / \mathrm{mL}$. Em todos os indivíduos tiroidectomizados em razão de carcinoma diferenciado de tiróide $(n=30)$, os níveis basais de calcitonina foram inferiores a $1 \mathrm{pg} / \mathrm{mL}$. Assim como em nosso laboratório, o valor de calcitonina considerado normal é menor que $10 \mathrm{pg} / \mathrm{mL}$. N ão temos acesso às informações clínicas de cada indivíduo, porém ressaltamos que, de acordo com a literatura, a hipótese de CM T deve ser considerada em pacientes com valores de calcitonina basal maiores que $10 \mathrm{pg} / \mathrm{mL}$ (13).

\section{Testes de estímulo para secreção de calcitonina} Além da dosagem de calcitonina basal, existe a possibilidade de realização de sua dosagem após testes específicos de estímulo (exemplos de secretagogos para calcitonina incluem cálcio e/ ou pentagastrina, TRH e omeprazol) (14-19). Especificamente em casos de hiperplasia de células $C$, que corresponde a uma fase que antecede a malignização tumoral, ou em fase precoce do CM T, as concentrações basais de calcitonina podem estar levemente elevadas ou ainda estar dentro dos valores de referência. T al limitação pode ser superada por meio do uso destes testes de estímulo para secreção de calcitonina.

0 teste com pentagastrina é amplamente utilizado para o diagnóstico de CM T e para a avaliação de cura cirúrgica em pacientes operados de CM T com níveis basais de calcitonina indetectáveis (15). Após coleta da amostra basal, administra-se pentagastrina $0,5 \mu \mathrm{g} / \mathrm{kg}$, via endovenosa, e procede-se à coleta de amostras após 2, 5, 10 e 15 min. É importante lembrar que a pentagastrina pode ter como efeitos colaterais flushing, sensação de queimação em face, desconforto epigástrico, náuseas, taquicardia, tontura e urgência em defecar. O s valores basais para adultos saudáveis do sexo masculino não devem ultrapassar $10 \mathrm{pg} / \mathrm{mL}$; para as mulheres saudáveis, o valor normal é até $5 \mathrm{pg} / \mathrm{mL}$.
U m valor de calcitonina após a administração de pentagastrina superior a $100 \mathrm{pg} / \mathrm{mL}$ em qualquer tempo do teste é altamente sugestivo de presença de hiperplasia de células $C$ ou de CMT. Valores máximos de calcitonina considerados normais para tal teste não devem ultrapassar 30 a $40 \mathrm{pg} / \mathrm{mL}$. Valores entre 40 e $100 \mathrm{pg} / \mathrm{mL}$ são compatíveis com uma zona de incerteza e indica-se a reavaliação deste teste após 1 ano, ou mesmo antes, dependendo do grau de suspeita diagnóstica. Para os testes de estímulo com cálcio (gluconato de cálcio $2 \mathrm{mg} / \mathrm{Kg}$ de peso, via endovenosa) ou com cálcio associado à pentagastrina, os mesmos valores de referência devem ser considerados (16). O s testes com outros secretagogos, como TRH e omeprazol, são pouco utilizados rotineiramente.

\section{Ensaios para dosagem de calcitonina}

É importante destacar algumas características relacionadas aos ensaios para dosagem de calcitonina. U m detalhe importante em qualquer estratégia que faça uso de dosagem de calcitonina para tomadas de decisão envolvendo doença nodular tiroideana diz respeito à sensibilidade e especificidade do ensaio utilizado. Neste sentido, tratando-se de um marcador tumoral, as dosagens periódicas de calcitonina para seguimento de pacientes portadores de CMT devem ser realizadas preferencialmente utilizando-se 0 mesmo ensaio ou ensaios comparáveis do ponto de visa metodológico.

O s primeiros ensaios para dosagem de calcitonina eram radioimunoensaios, que, além da calcitonina monomérica circulante, reconheciam moléculas precursoras da calcitonina e seus metabólitos. Diversas formas de calcitonina imunorreativa estão presentes na circulação de indivíduos normais e de portadores de CM T. Estas formas diferentes de calcitonina circulantes podem ter peso molecular que varia de 3.400 (calcitonina monomérica) a $70.000 \mathrm{D}$ a (calcitonina polimérica) (20). $\mathrm{N}$ a dependência de fatores como diferenciação tumoral, as formas não monoméricas de calcitonina podem elevar-se anormalmente e mais precocemente que a forma monomérica. Assim, em alguns pacientes portadores de CMT a dosagem de calcitonina por RIE pode estar elevada antes que qualquer alteração seja observada nos ensaios imunométricos (21).

Todos os estudos mencionados anteriormente utilizaram ensaios imunométricos com dois anticorpos monoclonais com elevada sensibilidade (em torno de $2 \mathrm{pg} / \mathrm{mL}$ ) e especificidade para o monômero de calcitonina. Assim, em pacientes com doença nodular de tiróide, é mandatória a utilização de ensaios imunométricos para dosagem de calcitonina com um 
valor de cutoff menor ou igual a $10 \mathrm{pg} / \mathrm{mL}$, independentemente do uso de anticorpos monoclonais ou policlonais. D esta forma, como já foi dito, a hipótese de CMT deve ser considerada em pacientes com valores de calcitonina maiores que $10 \mathrm{pg} / \mathrm{mL}$. Em casos de elevação discreta do nível de calcitonina, recomenda-se a aplicação de um teste de estímulo e/ ou repetição da dosagem utilizando-se outro ensaio.

A incidência de elevações não específicas dos níveis de calcitonina parece ser menor nos ensaios que fazem uso de anticorpos monoclonais quando comparados aos ensaios que utilizam anticorpos policlonais. Insuficiência renal pode estar associada a valores elevados de calcitonina em alguns casos e níveis elevados de pró-calcitonina podem levar a reatividade cruzada com a calcitonina a ser dosada $(13,22,23)$. 0 aumento dos níveis basais de calcitonina em vigência de insuficiência renal é conhecido, e possivelmente está relacionado ao diminuído clearance renal de calcitonina observado nestes pacientes (24). A pró-calcitonina (PCT) geralmente não é secretada para a circulação. D esta forma, os níveis plasmáticos de PCT são muito baixos em indivíduos saudáveis. Entretanto, os níveis de PCT podem aumentar significativamente em condições como sepsis. $\mathrm{N}$ estes casos, alguns ensaios para medida de calcitonina podem apresentar reatividade cruzada com a PCT (11).

Recentemente, Saller e cols. (25), estudando pacientes com CMT, relataram um método para detecção em sangue periférico de células circulantes produtoras de calcitonina via RT-PCR e concluíram que esta metodologia, ainda que necessite de dados confirmatórios em número maior de pacientes, pode refletir a agressividade tumoral, podendo ser útil para a identificação precoce de doença disseminada e rapidamente progressiva.

Lembramos que, apesar da calcitonina ser reconhecidamente um marcador tumoral para CMT, outras doenças podem ser acompanhadas de elevação dos níveis séricos de calcitonina, tais como carcinoma pulmonar, síndrome carcinóide, carcinoma de mama e outras (26).

Em conclusão, nível de calcitonina basal superior a $10 \mathrm{pg} / \mathrm{mL}$ em ensaio imunométrico pode ser sugestivo de hiperplasia de células $\mathrm{C}$ ou mesmo CM T, principalmente em casos suspeitos para tais condições. Em pacientes portadores de nódulos de tiróide sem suspeita clínica prévia, valores de calcitonina basal acima do valor de referência devem ser valorizados e investigados, alternativamente com dosagem de calcitonina basal por outro método, de maneira a afastar possíveis interferentes, e/ ou aplicação de teste de estímulo para secreção de calcitonina.

\section{REFERÊNCIAS}

1. Zaidi M, Inzerillo AM, Moonga BS, Bevis PJ R, Huang CLH. Forty years of calcitonin - where are we now? A tribute to the work of lan Macintyre, FRS. Bone 2002;30:655-63.

2. Brandi ML, Gagel RF, Angeli A, Bilezikian JP, Beck-PeccozP, Bordi $C$, et al. Consensus: guidelines for dia gnosis and therapy of MEN type 1 and type 2. J Clin Endocrinol Metab 2001;86:5658-71.

3. Puñales MK, Graf H, Gross J L, Maia AL. Rastreamento genético do carcinoma medular de tireóide: identificação de mutações no proto-oncogene Ret. Arq Bras Endocrinol Metab 2002;46:632-9.

4. Wohllk N, Cote GJ , Bugalho MM, et al. Relevance of RET proto-oncogene mutations in sporadic medullary thyroid carcinoma. J Clin Endocrinol Metab 1996;81:3740-5.

5. Pacini F, Fontanelli M, Fugazzola L, et al. Routine measurements of serum calcitonin in nodular thyroid diseases allows the preoperative diagnosis of unsuspected sporadic medullary thyroid carcinoma.J Clin Endocrinol Metab 1994;76:826-9.

6. Dunn JT. Editorial: When is a thyroid nodule a sporadic medullary carcinoma? J Clin Endocrinol Metab 1994; 76:824-5.

7. Nic coli $\mathrm{P}$, Wion-Barbot $\mathrm{N}, \mathrm{C}$ aron $\mathrm{P}$, et al. Interest of routine measurement of serum calcitonin: study in a large series of thyroidectomized patients. J Clin Endocrinol Metab 1997;82:338-41.

8. Horvit PK, Gagel RF. Editorial: The goitrous patient with an elevated serum calcitonin - what to do? J Clin Endocrinol Metab 1997;82:335-7.

9. Rieu M, Lame MC, Richard A, et al. Prevalence of sporadic medullary thyroid carcinoma: the importance of routine measurement of serum calcitonin in the diagnostic evaluation of thyroid nodules. Clin Endocrinol 1995;42:453-60.

10. Ozgen AG, Hamulu F, Bayratkar F, et al. Evaluation of routine basal serum calcitonin measurement for early diagnosis of medullary thyroid carcinoma in seven hundred seventy-three patients with nodular goiter. Thyroid 1999;9:579-82.

11. Cohen R, Campos J M, Salaün $C$, et al. Pre-operative calcitonin levels are predictive of tumor size and postoperative calcitonin nomalization in medullary thyroid carcinoma. J Clin Endocrinol Metab 2000;85:919-22.

12. Grauer A, Raue F, Zegler R. Clinic al usefulness of a new chemiluminesc ent two-site immunoassa y for human calcitonin. Exp Clin Endocrinol Diabetes 1998;106:353-9.

13. d'Herbonnez M, Leclerc L, Vantyghem MC, Foumier F, Proye C, Wemeau J L. Clinical evaluation of a new sensitive calc itonin assay: study of spec ific ity. Clin Chim Acta 2001;311:149-55.

14. Maciel RMB. O laboratónio no diagnóstico e seguimento de doenças auto-imunes e neoplásicas de tiróide. Arq Bras Endocrinol Metab 2002;46:65-71.

15. Vitale G, Ciccarelli A, Caraglia M, et al. Comparison of two provocative tests for calcitonin in medullary thyroid carcinoma: omeprazole vs. pentagastrin. Clin Chem 2002;48:1505-10. 
17. Vieira JGH, Maciel RMB, Hauache OM. Manual de provas funcionais em endocrinologia e metabologia. Fleury - Centro de Medic ina Diagnóstica, 2001.

18. Guilloteau D, Perdrisot R, Calmettes C, et al. JC. Diagnosis of medullary carcinoma of the thyroid (MCT) by calcitonin assay using monoclonal antibodies: criteria for the pentagastrin stimulation test in hereditary MCT. J Clin Endocrinol Metab 1990;71:1064-7.

19. Barbot N, Calmettes C, Schuffenechker I, et al. Pentagastrin stimulation test and early diagnosis of medullary thyroid carcinoma using an immunoradiometric as of calcitonin: comparison with genetic screening in hereditary medullary thyroid carcinoma. J Clin Endocrinol Metab1994; 78:114-20.

20. Scheuba C, Kaserer K, Weinshäusl A, et al. Is medullary thyroid cancer predictable? A prospective study of 86 patients with abnomal pentagastrin tests. Surgery 1999;126:1089-96.

21. Body JJ, Heath III H. Estimates of circulating monomeric calcitonin: physiological studies in nomal and thyroidectomized man. J Clin Endocrinol Metab 1983;57:897-903.

22. Abelin N, GomesS, Iva noff MT, Ezabella MCL, Hayashida CY, Toledo SPA. Ábordagem clínica e laboratorial do bócio uninodular sólido: vantagem da deteminação da calcitonina sérica por métodos distintos. Arq Bras Endocrinol Metab 1999;43:104-13.

23. Engelbach M, Görges R, Forst T, et al. Improved diagnostic methods in the follow-up of medullary thyroid car- cinoma by highly specific calcitonin measurements. J Clin Endocrinol Metab 2000;85:1890-4.

24. Saller B, Görges R, Reinhardt W, Haupt $K$, Janssen $O$, Mann K. Sensitive calcitonin measurement by two-site immunoradiometric assays: implications for calcitonin screening in nodular thyroid disease. Clin Lab 2002; 48:191-200.

25. Niccoli $P$, Brunet $P$, Roubicek $C$, et al. Abnomal calcitonin basal levels and pentagastrin response in patients with chronic renal failure on maintenance hemodialysis. EurJ Endocrinol 1995;132:75-81.

26. Saller B, Feldmann G, Haupt K, et al. RT-PCR-based detection of circulating calcitonin-producing cells in patients with advanced medullary thyroid cancer. J Clin Endocrinol Metab 2002;87:292-6.

27. Friedman RB, Young DS. Effects of disease on clinical laboratory tests. $3^{\text {rd }}$ ed. Washington: AACC Press; 1997.

\section{Endereço para comespondência:}

Omar M. Hauache

Setor de Endocrinologia - Fleury -

Centro de Medicina Diagnóstica

Rua General Waldomiro de Lima 508

04344-070 São Paulo, SP

Fax: (011) 5014-7425

e.mail: omar.hauache@fleury.com.br 